\title{
L'État comme personne et comme représentation ou les jeux de miroir de la légalité et de la légitimité
}

The State as Person and as Representative, or the Mirror Image of Legality and Legitimacy

Éric Maulin

\section{(2penEdition Journals}

Édition électronique

URL : https://journals.openedition.org/cps/2261

DOI : 10.4000/cps.2261

ISSN : 2648-6334

Éditeur

Presses universitaires de Strasbourg

\section{Édition imprimée}

Date de publication : 1 juin 2012

Pagination : 171-184

ISBN : $978-2-354100-44-5$

ISSN : $1254-5740$

\section{Référence électronique}

Éric Maulin, «L'État comme personne et comme représentation ou les jeux de miroir de la légalité et de la légitimité », Les Cahiers philosophiques de Strasbourg [En ligne], 31 | 2012, mis en ligne le 15 mai 2019, consulté le 08 novembre 2022. URL : http://journals.openedition.org/cps/2261 ; DOI : https:// doi.org/10.4000/cps.2261

\section{(c) (i) (-)}

Creative Commons - Attribution - Pas d'Utilisation Commerciale - Partage dans les Mêmes Conditions 4.0 International - CC BY-NC-SA 4.0

https://creativecommons.org/licenses/by-nc-sa/4.0/ 


\section{L'État comme personne et comme représentation ou les jeux de miroir de la légalité et de la légitimité}

Éric Maulin

La définition de l'État comme une personne juridique est une définition courante, technique, fonctionnelle: elle permet d'imputer à une entité abstraite un patrimoine, un intérêt, une responsabilité, une souveraineté. Cette apparente banalité résulte surtout de la généralité d'un usage. Elle est pourtant grosse d'un anthropomorphisme impensé qui nous met en présence d'une de ces fictions que les juristes affectionnent. Comment peut-on dire de l'État, "le plus froid des monstres froids", qu'il est une personne, qu'il a une personnalité et présente ainsi une ressemblance au moins analogique avec la personne humaine? Qu'est-ce que cette qualification recouvre? Comment est-elle apparue et comment la comprendre?

L'étrangeté du recours à ce grossier anthropomorphisme pour expliquer la volonté de l'État et dissimuler sous cette volonté générale et abstraite la volonté particulière et intéressée des gouvernants a été très souvent dénoncée. Le publiciste Léon Duguit, au début du XXe siècle, a écrit tout un ouvrage, dans un esprit positiviste, pour démasquer cette métaphysique toute germanique, selon lui, qui se tapissait à l'ombre de la personnalité de l'État ${ }^{1}$. Sans la métaphysique de la personnalité, l'État, nu, n'apparaît que dans les dires et les actions d'individus qui s'agitent et décident en l'invoquant sans cesse sans le faire apparaître jamais. Cette critique prouve-t-elle que le recours à la personnalité de l'État est faux ou bien, au contraire, que la fiction est nécessaire pour faire apparaittre

1 Léon Duguit, L'État, le droit objectif et la loi positive, Paris, Fontemoing, 1901 (réimpression Dalloz, 2003); L'État, les gouvernants et les agents, Paris, Fontemoing, 1903 (réimpression Dalloz, 2005). 
l'État? La personnalité de l'État est un peu comme le corps mystique de l'Église: une réalité invisible et cependant constitutive de l'essence même de l'institution. Oublier que l'Église est le corps mystique du Christ, écrivait Pie XII dans la lettre encyclique Mystici corporis Christi ${ }^{2}$, c'est oublier le sens même de l'institution ecclésiastique pour ne plus la penser que comme une réalité sociologique, juridique ou morale. Risquons ici une analogie: dépouiller l'État de sa personnalité revient à s'empêcher de penser ce qui fait son essence même, à savoir la souveraineté, pour ne plus le comprendre que sous l'action de ses gouvernements et gouvernants successifs. La métaphysique de la personnalité de l'État n'est pas ce qui dissimule la réalité du pouvoir exercé par les acteurs - car en un sens purement sociologique, le pouvoir n'existe pas, il n'y a nul lieu du pouvoir - mais elle est la construction intellectuelle et la fiction de sa personnalité qui rend intelligible l'État et permet de le penser comme un acteur.

\section{Personnalité de l'État et souveraineté}

De nombreuses entités se voient reconnaître la personnalité juridique: les associations, les entreprises, les établissements publics, les collectivités territoriales. Selon leurs natures ou leurs fonctions, elles sont des personnes de droit privé ou de droit public. Le fondement de la personnalité de ces entités a toujours été discuté par les juristes: est-ce de par la loi qu'elles se voient octroyer la personnalité, habilitées à agir comme des sujets de droit? Ou est-ce, au contraire, en raison de leur utilité sociale qu'elles se voient reconnaître une personnalité en quelque sorte préexistante? Ces questions âprement débattues autrefois sont aujourd'hui tombées dans l'oubli: on reconnaît la personnalité à toute entité à laquelle on veut affecter un patrimoine ou imputer une responsabilité. Cette reconnaissance procède de la loi, de la déclaration, de la rédaction de statuts, conformément à la loi ou aux principes dégagés par la jurisprudence. Si le fondement de la personnalité collective n’est

2 Mystici Corporis Christi, Lettre encyclique de Sa Sainteté, le Pape Pie XII, sur le Corps mystique de Jésus-Christ et sur notre union en lui avec le Christ, datée du 29 juin 1943, accessible sur le site du Saint-Siège, http://www.vatican.va/holy_father/pius_xii/encyclicals/documents/hf_pxii_enc_29061943_mystici-corporis-christi_fr.html 
plus vraiment discuté, la fiction de la personnalité étend loin son empire. Le code pénal français consacre ainsi aujourd'hui la responsabilité pénale des personnes morales, autrement dit leur impute non seulement une responsabilité civile, donc pécuniaire, mais une responsabilité pénale, donc morale: la personne morale peut non seulement mal faire mais faire le mal.

Une personne échappe cependant à cette responsabilité pénale, c'est l'État. L'État peut mal faire, et c'est pourquoi le principe de la responsabilité civile de l'État et de ses agents est depuis longtemps consacré (il est contemporain de l'institution de la République en France), mais il ne peut faire le mal, et cela parce qu'il est souverain. La doctrine de la responsabilité de protéger ${ }^{3}$, développée au sein de l'Organisation des Nations unies, nous explique que lorsque les gouvernants font le mal, par exemple massacrent la population civile, ils ne sont plus les gouvernants, ils n'incarnent plus l'État, c'est-à-dire qu'ils n'agissent plus au nom de la souverainetét. Ils sont des particuliers, comme une bande de brigands, usant d'une force de contrainte qui relève du droit commun et qu'une opération de police internationale peut arrêter. Cette exception concernant l'État, qui est une personne mais n'est pas moralement (dans le sens de "éthiquement») responsable, a sa raison d'être.

Parmi les personnes juridiques l'une se détache en raison de sa spécificité, c'est l'État. L'État n'est pas seulement une personne juridique, c'est une personne juridique souveraine. C'est la souveraineté qui fait l'être de l'État, comme le dit Bodin en filant une classique métaphore nautique:

«Tout ainsi que le navire n'est plus que bois, sans forme de vaisseau, quand la quille qui soutient les côtés, la proue, la poupe et le tillac sont

3 La responsabilité de protéger est la doctrine formulée dans l'enceinte de l'Organisation des Nations unies à partir de 2005 et destinée à donner un cadre légal international au droit d'intervention humanitaire sans verser dans l'ingérence.

4 Après avoir défini l'État comme "un droit gouvernement de plusieurs mesnages et de ce qui leur est commun avec puissance souveraine», Bodin ajoute: "Nous avons dit en premier lieu, droit gouvernement, pour la différence qu'il y a entre les Républiques, et les troupes de voleurs et pirates, avec lesquels on ne doit avoir part, ny commerce, ny alliance: comme il a tousjours été gardé en toute République bien ordonnée» (Jean Bodin, Les six livres de la République (1593), Paris, Fayard, $10^{e}$ éd., 1986, I, 2, p. 41). 
ôtés: aussi la République sans puissance souveraine, qui unit tous les membres et parties d'icelle, et tous les ménages et collèges en un corps, n'est plus République..." ${ }^{5}$

L'État n'est pas seulement une personne mais une personne souveraine, et c'est la souveraineté qui tient ensemble ses constituants. La souveraineté, dans l'État, n'est pas au monarque, au président, au gouvernement, au parlement, ni à n'importe quel organe, ni même au peuple en ce qu'il constituerait un être en soi, une personne, mais elle est à l'État considéré comme une personne (par exemple, la personnification de la nation, ou du peuple), autrement dit, elle n'est à personne. C'est un point sur lequel de nombreux juristes ont mis l'accent: dire que l'État est une personne juridique souveraine revient à dire que la souveraineté n'est pas la propriété d'un individu ou d'un groupe d'individus, une puissance détenue en propre par tel ou tel particulier, mais que la souveraineté est la propriété d'un système juridique. Entre ces deux sens du mot "propriété», il y a pourtant plus qu'une nuance: la propriété de la souveraineté n'est pas ici un avoir mais un être. L'État n'a pas la souveraineté comme une propriété qu'il pourrait vendre, aliéner, débiter par morceaux, mais il est souverain. De cet être résultent les qualités secondes que l'on prête à la souveraineté: indivisibilité, inaliénabilité, perpétuité. La souveraineté de la personne État est la traduction de la dépatrimonialisation du pouvoir.

Définir l'État souverain comme une personne juridique est alors équivalent à dire que tout État est une République, autrement dit que le pouvoir n'est à personne, que le lieu de la souveraineté, comme l'écrivait Lefort, est un lieu vide, que l'exercice du pouvoir légal n'est jamais que l'exercice d'une compétence constitutionnellement dévolue à une autorité précaire au nom de l'État selon une chaîne d'habilitations juridiques. L'acte légal d'autorité se donne comme une figure de style: c'est la prosopopée. La justice est rendue, en France, «au nom du peuple français", lequel n'est pas dans la rue ni même dans les urnes, mais dans la représentation institutionnelle qui en est constamment ordonnée.

En ce sens, la qualification de l'État comme personne juridique, loin d'apparaître comme une forme d'anthropomorphisme qui imputerait à des entités abstraites des formes et des volontés humaines, est comprise, au contraire, comme un moyen d'arracher la puissance publique à des

\section{Ibidem.}


volontés humaines particulières tentées de se l'approprier en vue de la conservation de leurs propres intérêts particuliers, pour enserrer cette puissance publique dans un cadre juridique et la redistribuer sous la forme de dévolution de compétences. Dans un État de droit, en effet, les organes de l'État, les gouvernants, les fonctionnaires, les agents de l'État n'exercent pas à proprement parler un pouvoir mais seulement une compétence. C'est-à-dire qu'ils ne sont pas propriétaires du pouvoir qu'ils exercent mais seulement mandataires.

On peut illustrer cette thèse en mentionnant l'article 3 de la Constitution de 1958. «La souveraineté nationale appartient au peuple qui l'exerce par ses représentants et par la voie du référendum ", dispose cet article. "Aucune section du peuple ni aucun individu ne peut s'en attribuer l'exercice." Deux observations peuvent être formulées: premièrement, la titulature de la souveraineté et son exercice sont nettement dissociés ("appartenir»/ "exercer»), ce qui traduit bien le fait que ceux qui exercent la souveraineté ne la possèdent pas et en sont encore moins propriétaires. Ils l'exercent «au nom de...»; ici, «au nom du peuple». Deuxièmement, le peuple lui-même, titulaire de la souveraineté, n'existe pas absolument, il n'existe pas en soi. Il existe «des gens", la population, "des français", mais aucune de ces expressions ne renvoie au principe d'une unité d'intention, de volonté et d'action. Le peuple ne veut qu'au travers des procédures institutionnelles qui expriment une volonté pour son compte, qui expriment $s a$ volonté, selon un processus d'imputation qui reste à préciser.

On peut comprendre alors que la souveraineté est exercée ici par les organes, des agents, des gouvernants, des représentants, de l'État, qui n'exercent que des fonctions et des compétences. En revanche le peuple lui-même ne peut vouloir que par le moyen de ses organes étatiques. Il n'est ontologiquement pas en mesure de vouloir autrement qu'au travers des mécanismes institutionnels de sa représentation.

Cette idée est souvent rendue par la définition, devenue canonique, en France, mais souvent mal comprise: «l'État est la personnification juridique de la nation $»^{6}$. Car cette formule veut dire, rigoureusement, le contraire de ce qu'elle semble dire: elle veut dire que la souveraineté, loin

6 Adhémar Esmein, Éléments de droit constitutionnel, Paris, Larose, 1894; Raymond Carré de Malberg, Contribution à la théorie générale de l'État, t. 1, Paris, Sirey, 1921. 
d'être incarnée par une figure concrète de l'exercice du pouvoir, est au contraire entièrement dépersonnalisée: elle n'est nulle part ailleurs que dans le système juridique national constitutif de l'État.

\section{Dépersonnalisation du pouvoir et représentation}

La définition de l'État comme personne juridique a pour conséquence de dépersonnaliser la souveraineté. Cette dépersonnalisation du pouvoir qui résulte de la qualification de l'État comme personne juridique est une préoccupation ancienne. On peut essayer de tracer la genèse de cette idée en montrant comment le passage d'un principe de la souveraineté monarchique à un principe de souveraineté nationale ou populaire a obligé à en repenser le concept et permis l'invention de cette idée de personnalité de l'État.

De nombreux légistes ont essayé de penser la dissociation de l'exercice du pouvoir par le roi envisagé comme un individu concret, un corps mortel, de sa titulature par le roi compris comme une institution? Ils ont distingué pour cela entre deux corps du roi: le corps mortel, soumis à l'erreur et à la corruption, qui est le corps de l'individu, du sujet mortel, et le corps politique, surnaturel, immortel, ne se trompant jamais, dépourvu de faiblesse, incarnant le royaume. Ces théories ont été analysées en détail par les cérémonialistes américains, en premier lieu Ernst Kantorowicz, dans Les deux corps du roi, et Ralph E. Giesey, dans Le roi ne meurt jamais: étude du cérémonial funèbre des rois de France à l'époque de la Renaissance.

La fonction de ces constructions savantes est de mettre en évidence la manière dont la souveraineté, parfaite, impérissable, est soustraite dans

7 Citons ici Jean de Terrevermeille, dans son Tractatus de jure futuri successoris legitimi in regiis hereditatibus, 1419 , et le commentaire autorisé de Jean Barbey, La Fonction royale. Essence et légitimité d'après les Tractatus de Jean de Terrevermeille, Paris, Nouvelles éditions latines, 1983. Et beaucoup plus tard, refermant en quelque sorte la théorie des deux corps, Charles Loyseau, Traité des Offices, 1614, et le commentaire autorisé de Brigitte BASDEVANT, Aux origines de l'État moderne: Charles Loyseau (1564-1627), théoricien de la puissance publique, Paris, Economica, 1984.

8 Ernst Kantorowicz, Les deux corps du roi: essai de théologie politique au Moyen Age (1957), trad. fr. J.-Ph. Genet et N. Genet, Paris, Gallimard, 1989; Ralph E. GIESEY, Le roi ne meurt jamais : étude du cérémonial funèbre des rois de France à l'époque de la Renaissance, Paris, Flammarion, 1992. 
son principe à la finitude de l'exercice d'un pouvoir par un être corruptible et mortel pour être imputé à son double incorruptible et immortel.

Dissertant sur la couronne impériale, Balde (1327-1406) insiste sur sa double nature. Posée sur la tête de l'empereur par le pape, la couronne est d'abord matérielle et visible. Mais elle aussi immatérielle et invisible en ce qu'à travers le pape, elle est posée par Dieu?. Une distinction analogue sera faite pour les monarchies. C'est autour de cette distinction que s'opère progressivement en Angleterre d'abord, en France ensuite, le lent phénomène de dépatrimonialisation du pouvoir.

L'un des premiers auteurs français à étudier cette question, dans un contexte polémique, celui de la succession de Charles VI, est Jean de Terrevermeille, un grand légiste du $\mathrm{XV}^{\mathrm{e}}$ siècle, attaché principalement à défendre les droits du futur Charles VII au trône de France après son exhérédation par son père, Charles VI. Dans le Tractatus $(1419)^{10}$, Jean de Terrevermeille s'attache principalement à démontrer que Charles VI ne pouvait déshériter le dauphin car il ne dispose pas de la Couronne comme d'une propriété féodale. Pour y parvenir, il distingue nettement entre la succession de droit privé et la succession de droit public, l'héritage patrimonial et la dévolution statutaire de la Couronne. Le cœur de son argumentation est de soutenir que Charles VI ne peut pas exhéréder son fils car il ne dispose pas de la Couronne comme d'une propriété. Il est sans doute le monarque souverain aussi longtemps qu'il est en vie mais il ne dispose pas de la souveraineté qui est la souveraineté de la Couronne. Son argumentation repose sur une interprétation de la loi salique et la distinction de la succession féodale et de la succession royale. Cette argumentation marque une étape importante dans l'histoire de la construction de l'État moderne en France. C'est en effet la perpétuité de la souveraineté de la Couronne, par-delà les contingences de son exercice séculier, qui se trouve formulée. À l'horizon de cette doctrine se profile l'État, comme entité permanente qui a pour fin le bien commun et qui dépasse les intérêts particuliers non seulement des sujets mais aussi du monarque. C'est la monarchie comme République.

Cette doctrine est développée par un légiste qui se situe à la charnière $\mathrm{du} \mathrm{XVI}^{\mathrm{e}}$ et du XVII ${ }^{\mathrm{e}}$ siècle, Charles Loyseau, dans son Traité des offices

9 Balde, Consilia, III, 159, n. 2, fol. 45v․ Cité par E. Kantorowicz, op. cit., p. 242-243 et p. 508 n. 76.

10 Voir note 7. 
(1614). S'inscrivant dans la droite ligne des Six livres de la République de Jean Bodin, Loyseau ajoute une précision sur laquelle l'auteur angevin n'avait pas insisté: il attribue explicitement la souveraineté à la Couronne (et non plus au roi physique), c'est-à-dire à l'institution monarchique. Le roi physique, le roi mortel, remplit seulement la fonction de premier officier de la Couronne.

On a là, grossièrement résumées, deux étapes essentielles de la formation de cette idée, bien mise en évidence par Giesey à travers l'étude du cérémonial des funérailles royales en France, pour montrer que «le roi ne meurt jamais». Mais si le roi ne meurt jamais, c'est finalement parce que le principe monarchique réside non dans le corps physique du monarque mais dans son corps politique, symbolisé par la couronne, et présentant cette double caractéristique d'être à la fois indivisible et perpétuelle.

Cette construction, ce dédoublement du corps du roi, n'est cependant pensable qu'à condition de pouvoir préalablement s'appuyer sur l'unité physique du corps royal. Elle devient inopérante à partir du moment où le titulaire de la souveraineté n'est plus le roi mais une entité collective, comme le peuple, car le point d'appui physique, le corps physique du roi, à partir duquel peut se construire l'analogie corporative, est naturellement impossible lorsque le souverain est une collectivité ou une pluralité, le peuple. À la différence du monarque, le peuple n'a pas d'unité corporative, il ne fait pas corps par lui-même. Il ne peut être qualifié de souverain qu'à condition, dans le même temps, d'être constitué en unité, ce qui est en apparence contradictoire: comment l'autorité instituante peut-elle d'abord être une autorité instituée? C'est ici qu'apparaît un mécanisme nouveau qui permet de comprendre la formation de cette unité: la représentation, ou plutôt, nous le verrons, la représentation en son sens moderne.

Le peuple, à la différence du monarque, n'a pas d'unité. Afin de répondre au problème de l'unité du peuple, Hobbes s'efforce de montrer comment la représentation en produit la focalisation et donc l'unification dans la personne du Léviathan, c'est-à-dire de l'État ${ }^{11}$. La définition de

11 Tout particulièrement dans le Léviathan ou traité de la matière, de la forme et du pouvoir d'une république ecclésiastique et civile (1651), trad. fr. F. Tricaud, Paris, Sirey, 1971. 
l'État comme personne juridique remplace ainsi la théorie des deux corps du roi pour penser la permanence de la souveraineté.

Avec Hobbes, on voit apparaitre l'idée que le détenteur du pouvoir n'agit pas en son propre nom mais au nom du peuple. Or agir au nom du peuple est précisément un trait caractéristique des institutions des États modernes. L'expression «au nom du peuple français», par exemple, est une expression juridique actuelle. La plupart des décisions de justice sont rendues "au nom du peuple français». Les paroles ou les actions de qui s'exprime au nom du peuple peuvent alors être reconnues comme les leurs par les membres de la communauté.

La Constitution américaine (et de nombreuses constitutions inspirées par elle, de manière analogue) commence par la formule "Nous, le peuple des États-Unis, afin de former une union plus parfaite, d'établir la justice, d'assurer la tranquillité intérieure, de pourvoir à la défense commune, de développer le bien-être général et d'assurer à nous-mêmes et à notre postérité les bienfaits de la liberté, ordonnons et établissons la présente Constitution pour les États-Unis d'Amérique». Cette magnifique prosopopée est le fait de représentants, à la Convention de Philadelphie, qui parlent si bien au nom du peuple qu'ils s'effacent pour le faire parler à travers eux ${ }^{12}$.

Un autre exemple, contemporain, est cette formule lâchée par Sieyès, lors de la transformation des États généraux en Assemblée nationale, le 17 juin 1789, qui veut que désormais l'assemblée nationale soit la nation assemblée. L'hypallage (puisqu'on est dans les figures de style de la représentation) inscrit l'assemblée nationale au plus près de la nation de telle sorte que l'on pense que c'est la nation elle-même qui s'exprime.

À l'occasion des deux événements fondateurs de l'État moderne, la révolution américaine et la révolution française, on constate que le souverain putatif, le peuple, la nation, ne peut s'exprimer qu'au moyen d'une représentation, c'est-à-dire d'une parole qui s'exprime en son nom. Or le grand théoricien de la représentation politique moderne a été Thomas Hobbes, et c'est naturellement dans son ouvre qu'il faut rechercher les fondements de ce mécanisme. Hobbes n'a pas seulement

12 On se reportera ici aux ouvrages de Bernard Manin, Principes $d u$ gouvernement représentatif, Paris, Flammarion, 1986; Didier Mineur, Archéologie de la représentation politique: structure et fondement d'une crise, Paris, Presses de la Fondation nationale des sciences politiques, 2010. 
été le théoricien de la représentation en ce sens que les gouvernants s'expriment au nom du peuple, mais il a surtout montré que ce peuple invoqué comme fondement de la parole souveraine n'existe cependant pas antérieurement à sa représentation : c'est l'acte même de sa représentation qui le constitue comme unité. Le paradoxe de la représentation politique est qu'elle constitue cela même qu'elle représente, puisque...

«avant l'accord de chacun avec chacun et la réunion de la multitude en une seule personne ou en une seule assemblée souveraine, il n'y a pas plus de peuple qu'il n'y a de souverain : que celui qui est fait souverain ne fasse pas de convention avec ses sujets avant son avènement, la chose est claire; $[. .$.$] avec l'ensemble constituant une partie contractante, cela$ est impossible, parce qu'ils ne sont pas encore une seule personne $»^{13}$.

La représentation politique est susceptible de prendre deux sens assez éloignés l'un de l'autre. Dans son premier sens - c'est le mot même qui le dit - elle implique en principe une distinction et une hiérarchie: une distinction entre le représentant et le représenté et une hiérarchie du représenté, antérieur et supérieur à son représentant chargé de parler à sa place. C'est en ce sens que l'on parle de représentation lorsqu'une personne donne mandat à une autre personne pour la représenter. Le mandant se trouve en effet porteur d'un mandat qui doit exprimer la volonté du mandataire et ce mandat a un caractère impératif. Le mandant ne fait que répéter la volonté du mandataire.

Loriginalité de Hobbes est d'avoir fait du concept de représentation un concept fondamental de sa pensée politique mais en inversant le rapport du représentant et du représenté. Confronté au problème de la réalité physique du peuple, sa dispersion, l'impossibilité de le saisir, Hobbes conçoit la représentation non comme le mécanisme qui va permettre de répéter une volonté populaire antérieure mais comme le mécanisme qui va permettre au contraire de constituer cette volonté populaire. À une conception mimétique de la représentation comme répétition, il va substituer une conception poïétique de la représentation comme création. La représentation politique chez Hobbes remplit une

13 Didier Mineur, «Les partis politiques et la logique philosophico-juridique de la représentation politique", Raisons politiques, $\mathrm{n}^{\circ} 20$, novembre 2005, p. 89-109; cf. Pierre Brunet, «La notion de représentation sous la Révolution française", Annales historiques de la Révolution française, $\mathrm{n}^{\circ} 2$, 2002, p. $27-45$. 
véritable fonction ontologique, en ce qu'elle constitue l'unité même du peuple:

"Une multitude d'hommes devient une seule personne quand ces hommes sont représentés par un seul homme ou une seule personne, de telle sorte que cela se fasse avec le consentement de chaque individu singulier de cette multitude $»^{14}$.

Le rapport de représentation est le principe de l'unité de la multitude qui lui confère sa personnalité.

La conception de la représentation dégagée par Hobbes permet de penser la manière dont les révolutions américaine et française vont instituer le mécanisme de la représentation: non comme la répétition d'une volonté populaire antérieure à sa représentation, mais comme la constitution même de cette volonté au nom du peuple.

Mais la philosophie de Hobbes est importante aussi parce qu'elle rompt avec l'aristotélisme des conceptions sociales antérieures. Pour Bodin, l'unité sociale est encore le ménage ( la souveraineté est droit gouvernement de plusieurs ménages...»), selon Hobbes, elle est l'individu. La pensée de Hobbes rompt avec le monde des corps et des corporations naturelles au profit d'un monde d'individus. Or c'est ce monde d'individus que prennent en compte les révolutions américaine et française, soucieuses en premier lieu de «rappeler» les droits individuels mais, aussi, par là même, de déconstruire l'ordre ancien.

\section{Légalité et légitimité}

L'une des conséquences les plus importantes de cette conception moniste de la représentation est, en effaçant la distinction du représentant et du représenté, d'abolir la distinction de la légitimité et de la légalité. Dire que la volonté n'existe nulle part ailleurs qu'au lieu de sa représentation revient à dire qu'il n'y a pas de volonté nationale alternative à sa représentation. Que le corps électoral, à proprement parler, ne veut pas mais qu'il se contente d'élire et que l'élection est essentiellement distincte de la législation. C'est pour bien rendre cette conclusion, qui s'impose dès lors que l'on conçoit l'État comme la personnification juridique de la nation, qu'un certain nombre d'auteurs, à commencer par Carré de Malberg, proposent de substituer à l'idée de représentation

14 Th. Hоввеs, Léviathan, éd. cit., chap. XVI, p. 166. 
celle d'organe. Cette démarche exprime bien ce basculement, qui sera caractéristique de la III ${ }^{e}$ République, où la représentation du peuple souverain est en fait une représentation souveraine du peuple.

La volonté étatique, la loi, ne provient pas d'un mécanisme de représentation mais d'organisation. C'est-à-dire que les députés ne sont pas les représentants du peuple mais les organes de l'État. La volonté nationale n'est pas répétée par des représentants mais créée par des organes. Or une telle conception, sur le fondement du principe démocratique, n'est compréhensible que si la nation est assimilée à l'État, que si l'État est la personnification juridique de la nation. La définition de l'État comme personnification juridique de la nation est intimement liée à la théorie des organes de l'État.

Dans cette perspective, la représentation n'est plus l'expression ou la rationalisation d'une volonté antérieure de la collectivité, mais la production même de cette volonté. La collectivité, le peuple, ou la nation se confondent avec l'État, en ce sens que lui seul produit une volonté imputée ensuite à la collectivité. La représentation de la volonté collective par un certain nombre d'individus se présente à nous comme la constitution même de la volonté de l'État, élaborée en vertu d'une "concession constitutionnelle de puissance poussée jusqu'à un certain degré $»^{15}$. L'État nous apparaît ainsi comme un ensemble d'organes derrière lesquels il n'y a rien: il est l'unique réalité de l'unité de la collectivité. En ce sens, on peut conclure que «l'État ne peut exister que moyennant ses organes; si par la pensée on supprime les organes, il ne reste plus juridiquement que le néant ${ }^{16}$.

L'organe est l'autorité qui initie la volonté de l'État ou de la nation. Carré de Malberg donne un sens fort à cette expression. À proprement parler, l'organe ne fait pas qu'initier la volonté d'une personne «État» ou «nation" qui préexisterait à sa capacité de vouloir dans les confins d'un arrière-monde, mais il crée la personnalité du groupe qui, antérieurement, n'existait pas encore. Logiquement, la volonté de l'organe précède celle de la personnalité qu'elle constitue. Au contraire, les simples autorités ne sont pas des organes parce qu'elles ne sont pas indispensables à la formation de la volonté étatique, en ce sens que «la personnalité et la

15 Carré de Malberg, Contribution à la théorie générale de l'État, Paris, Sirey, 1921, t. II, p. 277.

16 Idem, t. I, p. 39-40. 
volonté de l'État se trouvent complètement formées, dès avant toute intervention de leur activité ${ }^{17}$.

C'est le propre du constitutionnalisme de ne plus accepter d'argumentation juridique $d u$ dehors. L'alternance politique, les évolutions de légitimité se construisent à l'intérieur d'un ordre de la légalité qui en est toujours la condition de possibilité. Qu'une pensée transcende cet ordre légal et elle reste inaudible, insaisissable en dehors du jeu institutionnel de l'État.

\section{Bibliographie}

BARbeY Jean, La Fonction royale. Essence et légitimité d'après les Tractatus de Jean de Terrevermeille, Préface de Marguerite Boulet-Sautel, Paris, Nouvelles éditions latines, 1983.

Basdevant Brigitte, Aux origines de l'État moderne: Charles Loyseau (1564-1627), théoricien de la puissance publique, Paris, Economica, 1984.

Bodin Jean, Les six livres de la République (1593), Paris, Fayard, 10éd., 1986.

BRUnet Pierre, "La notion de représentation sous la Révolution française», Annales historiques de la Révolution française, $\mathrm{n}^{\circ} 2$, 2002, p. 27-45.

Duguit Léon, L'État, le droit objectif et la loi positive, Paris, Fontemoing, 1901 (réimpression Dalloz, 2003).

Duguit Léon, L'État, les gouvernants et les agents, Paris, Fontemoing, 1903 (réimpression Dalloz, 2005).

Carré de Malberg Raymond, Contribution à la théorie générale de l'État, 2 volumes, Paris, Sirey, 1921.

Esmein Adhémar, Eléments de droit constitutionnel, Paris, Larose, 1894.

GIESEY Ralph E., Le roi ne meurt jamais : étude du cérémonial funèbre des rois de France à l'époque de la Renaissance, Paris, Flammarion, 1992.

Hoвbes Thomas, Léviathan ou traité de la matière, de la forme et du pouvoir d'une république ecclésiastique et civile (1651), trad. fr. F. Tricaud, Paris, Sirey, 1971.

Kantorowicz Ernst, Les deux corps du roi: essai de théologie politique au Moyen Age, trad. fr. J.-Ph. Genet et N. Genet, Paris, Gallimard, 1989 (éd. originale:1957).

17 Idem, t. II, p. 396. 
Manin Bernard, Principes du gouvernement représentatif, Paris, Flammarion, 1986.

Mineur Didier, "Les partis politiques et la logique philosophicojuridique de la représentation politique», Raisons politiques, n 20 , novembre 2005, p. 89-109.

Mineur Didier, Archéologie de la représentation politique: structure et fondement d'une crise, Paris, Presses de la Fondation nationale des sciences politiques, 2010.

Mystici Corporis Christi, Lettre encyclique de Sa Sainteté, le Pape Pie XII, sur le Corps mystique de Jésus-Christ et sur notre union en lui avec le Christ, datée du 29 juin 1943, accessible sur le site du Saint-Siège, http://www.vatican.va/holy_father/pius_ xii/encyclicals/documents/hf_p-xii_enc_29061943_mysticicorporis-christi_fr.html 